\title{
Effect of mild hypothermia on expression of inflammatory factors in surrounding tissue after minimally invasive hematoma evacuation in the treatment of hypertensive intracerebral hemorrhage
}

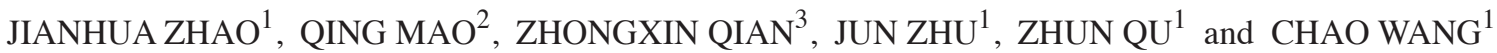 \\ ${ }^{1}$ Department of Neurosurgery, Shanghai Pudong New Area Gongli Hospital, Shanghai 200135; \\ ${ }^{2}$ Department of Neurosurgery, Shanghai Renji Hospital, Shanghai Jiaotong University, School of \\ Medicine; ${ }^{3}$ Department of Neurosurgery, Shanghai Punan Hospital, Shanghai 200127, P.R. China
}

Received December 14, 2017; Accepted February 16, 2018

DOI: $10.3892 / \mathrm{etm} .2018 .6014$

\begin{abstract}
Mild hypothermia combined with minimally invasive hematoma evacuation was evaluated in the treatment of hypertensive intracerebral hemorrhage to reduce inflammatory response of brain tissue around hematoma and ameliorate brain function, and to investigate its safety, effectiveness and feasibility. A total of 206 patients with acute spontaneous hypertensive intracerebral hemorrhage were collected clinically and randomly divided into minimally invasive hematoma evacuation group (group A) and mild hypothermia combined with minimally invasive hematoma evacuation (group B). The National Institutes of Health Stroke Scale (NIHSS) score was used before and after treatment. Group A was treated with minimally invasive intracranial hematoma evacuation using intracranial hematoma grinding puncture needle while group B received whole body water circulation type cooling blanket plus local cerebral mild hypothermia therapy with ice cap on the basis of minimally invasive surgery. Patients' brain tissue fragments around hematoma taken out with rinsing during operation and at postoperative 1, 3 and 7 days were investigated. The contents of tumor necrosis factor- $\alpha$ (TNF- $\alpha$ ) in serum at postoperative 1, 3 and 7 days were evaluated by enzyme-linked immunosorbent assay (ELISA). For the degree of nerve function defect of patients in the two groups, NIHSS score was lower in group B than that in group A at days 3 and 7, and the differences were statistically significant $(\mathrm{P}<0.05)$. The serum TNF- $\alpha$ content and expression of nuclear factor $-\kappa B$ $(\mathrm{NF}-\kappa \mathrm{B})$ in brain tissue around hematoma reached the peak on the 3rd day. The TNF- $\alpha$ content and NF- $\kappa \mathrm{B}$ expression were
\end{abstract}

Correspondence to: Dr Qing Mao, Department of Neurosurgery, Shanghai Renji Hospital, Shanghai Jiaotong University, School of Medicine, 160 Pujian Road, Shanghai 200127, P.R. China

E-mail: iqpps724@163.com

Key words: inflammatory response, intracerebral hemorrhage, minimally invasive hematoma evacuation, $\mathrm{NF}-\kappa \mathrm{B}$, mild hypothermia lower in group B than those in group A at each time-point $(\mathrm{P}<0.05)$. Mild hypothermia combined with minimally invasive hematoma evacuation can reduce the damage of hematoma to the surrounding brain tissue, effectively alleviate inflammatory response and decrease brain tissue injury, thus ameliorating brain function.

\section{Introduction}

Intracerebral hemorrhage is a common and frequently-occurring disease with high mortality and disability rates, accounting for $10-15 \%$ of cerebrovascular diseases, and the mortality rate is greater than $50 \%$ (1). The curative effect is directly affected by the therapeutic method after intracerebral hemorrhage. The minimally invasive hematoma evacuation early used for patients with hypertensive intracerebral hemorrhage can reduce the compression to surrounding brain tissue by hematoma, and ischemia and edema caused by local hematoma compression can be relieved; after hematoma is removed, the damage of brain tissue around hematoma caused by hematoma decomposition product can also be reduced, thus ameliorating brain function. Additionally, great attention has been paid to the neuroprotective effect of mild hypothermia therapy; it can protect brain tissue via inhibiting inflammatory response, reducing brain edema, decreasing metabolism of brain tissue $(2,3)$. In recent years, the study on intracerebral hemorrhage has gradually focused on brain function protection of brain tissues around the hematoma, generally considering that inflammatory response is involved in the pathological process of the damage of brain tissue around hematoma after intracerebral hemorrhage. In the early stage of intracerebral hemorrhage, inflammatory response already exist in the local brain tissue around the hematoma; therein, nuclear factor- $\mathrm{\kappa B}(\mathrm{NF}-\kappa \mathrm{B})$ and inflammatory cytokine, tumor necrosis factor- $\alpha$ (TNF- $\alpha$ ), play important roles, which can aggravate the damage of brain tissue around the hematoma after intracerebral hemorrhage. By comparing postoperative expression of NF- $\mathrm{kB}$ and TNF- $\alpha$ after treatment with mild hypothermia combined with minimally invasive hematoma evacuation or minimally invasive hematoma evacuation only, 
the present study aimed to investigate the protective effect of mild hypothermia therapy in reducing inflammatory damage after minimally invasive hematoma evacuation in the treatment of intracerebral hemorrhage.

\section{Patients and methods}

Clinical data. A total of 206 patients with acute spontaneous hypertensive intracerebral hemorrhage in Shanghai Renji Hospital (Shanghai, China) between May 2014 and September 2016 were collected. The patients, 111 males and 95 females, were treated within $48 \mathrm{~h}$ of onset, and they suffered the first onset at the average age of 55.67 \pm 21.53 years. This study was approved by the Ethics Committee of Shanghai Renji Hospital. Signed written informed consents were obtained from all participants before the study. All patients conformed to the Fourth Cerebrovascular Diseases Academic Meeting of the Chinese Medical Association: Diagnostic Points of Various Cerebrovascular Diseases and diagnosed as intracerebral hemorrhage by head computed tomography (CT) and magnetic resonance imaging (MRI). Calculation of hematoma volume after intracerebral hemorrhage: According to the CT film measurement and Coniglobus formula (volume $=\pi / 6 \mathrm{x}$ length $\mathrm{x}$ width $\mathrm{x}$ layer) calculation, the amount of bleeding was $>30 \mathrm{ml}$ (4). The National Institutes of Health Stroke Scale (NIHSS) score was used before and after treatment and examined by the same neurology physician. Patients were treated by minimally invasive hematoma evacuation on the day of onset or on the following day $(<48 \mathrm{~h})$. The mild hypothermia treatment group received local mild hypothermia therapy immediately after the operation, and the conventional therapies including dehydrant and brain protectants were taken by the two groups. Exclusion criteria: All of the following cases were excluded to avoid confusion of inflammatory markers: i) Patients with inflammatory disease within half a month; ii) patients who were accompanied by severe complications; iii) patients with cardiovascular diseases, trauma, surgery, age less than 18 years or pregnancy within 6 months; iv) patients using drugs, such as anticoagulants, corticosteroids and $\beta$-receptor blockers; v) patients with abnormal blood pressure, systolic pressure $>210 \mathrm{mmHg}$ or $<100 \mathrm{mmHg}$; diastolic pressure $>110 \mathrm{mmHg}$ or $<50 \mathrm{mmHg}$; and vi) patients or their families who refused surgery.

Minimally invasive hematoma evacuation and mild hypothermia therapy. YL-1 type disposable intracranial hematoma grinding puncture needle (produced by Beijing Wantefu Medical Apparatus, Co., Ltd., Beijing, China) was utilized to perform minimally invasive hematoma evacuation. Before the surgery, blood pressure was controlled below $160 / 100 \mathrm{mmHg}$. The surgery was conducted under local anesthesia. The whole body water circulation type cooling blanket plus local cerebral mild hypothermia therapy with ice cap was adopted. Sedatives were used for patients with shivers. The whole body water circulation type cooling blanket (P\&C-A type cooling blanket; Hengbang, Beijing, China) and -4 to $-2^{\circ} \mathrm{C}$ ice cap (HGT-200 II type; Beijing D\&W Medical Equipment Co., Ltd., Beijing, China) were utilized as superficial hypothermia treatment. The brain temperature was decreased to $32.5-34.5^{\circ} \mathrm{C}$, which was displayed by tympanic temperature. The brain temperature was replaced by tympanic temperature on the affected side. Brain temperature $=$ tympanic temperature $\pm 0.5^{\circ} \mathrm{C}$ was regarded as standard, and it was measured by OMRON infrared ear thermometer after operation, 2 times per day, followed by taking the highest value. The duration of mild hypothermia was 3-7 days. Rewarming method: After mild hypothermia was stopped, the rewarming by $0.5^{\circ} \mathrm{C}$ should be maintained for patients approximately every $12 \mathrm{~h}$, followed by natural rewarming of body temperature to $\sim 37^{\circ} \mathrm{C}$.

NIHSS score. The NIHSS score was used before and after treatment $(5,6)$. The full marks are 42 points. The degree of nerve function defect was divided into: Mild, NIHSS score $<7$ points; moderate, NIHSS score $7-15$ points; and severe, NIHSS $>15$ points.

Detection of $N F-\kappa B$ and $T N F-\alpha$. The hypertensive patients with cerebral hemisphere bleeding volume over $30 \mathrm{ml}$ treated by minimally invasive hematoma evacuation on the day or second days of intracelebral hemorrhage were selected. The brain tissue fragments around hematoma taken out with rinsing during operation and at postoperative 1, 3 and 7 days were collected and immediately fixed using formaldehyde, followed by keeping at normal temperature. Meanwhile, $2 \mathrm{ml}$ morning fasting venous blood was extracted, centrifuged and placed at $-40^{\circ} \mathrm{C}$.

Statistical analysis. The experimental results were treated by SPSS 17.0 software (version X; SPSS, Inc., Chicago, IL, USA). The numerical value was expressed as mean \pm standard deviation (SD). The independent sample t-test was used for the comparison between two groups. A $\mathrm{P}<0.05$ was considered to indicate a statistically significant difference.

\section{Results}

NIHSS scores in the two groups of patients. The basic data at admission in the two groups of patients are shown in Table I. There were no statistically significant differences in sex, age, average initial NIHSS score, blood pressure, bleeding amount and other indexes in patients between the two groups. NIHSS score was the highest at admission and on the 1st day after treatment, which was gradually decreased with the treatment; it was significantly lower in group B than that in group $\mathrm{A}$ at 3 and 7 days, and the differences were statistically significant $(\mathrm{P}<0.05)$ (Table II).

Dynamic changes of serum TNF- $\alpha$ in the two groups of patients. Radioimmunoassay kit for serum TNF- $\alpha$ in two groups of patients was purchased from Wuhan Boster Biological Technology, Co., Ltd. (Wuhan, China). The concentration in group A was the highest at 3 days after treatment, and rapidly decreased at 7 days with the treatment; the serum TNF- $\alpha$ concentration in group B was gradually decreased, which had no difference at day 1 between the two groups; the concentration was significantly lower in group B than that in group A at days 3 and 7, and the differences were statistically significant $(\mathrm{P}<0.05)$ (Table III). 
Table I. Basic data of patients at admission (mean \pm SD).

\begin{tabular}{lccc}
\hline Items & Group A $(\mathrm{n}=103)$ & Group B (n=103) & P-value \\
\hline Age (years) & $51.53 \pm 15.39$ & $52.87 \pm 14.40$ & 0.19 \\
Admission delay (h) & $4.39 \pm 1.98$ & $4.75 \pm 2.06$ & 0.83 \\
NIHSS score & $17.04 \pm 3.06$ & $16.79 \pm 2.56$ & 0.56 \\
Bleeding amount (ml) & $38.19 \pm 9.32$ & $39.02 \pm 10.74$ & 0.92 \\
Systolic pressure $(\mathrm{mHg})$ & $148.73 \pm 17.59$ & $145.94 \pm 15.64$ & 0.37 \\
Diastolic pressure $(\mathrm{mmHg})$ & $96.29 \pm 11.59$ & $88.65 \pm 14.60$ & 0.18 \\
Temperature $\left({ }^{\circ} \mathrm{C}\right)$ & $37.26 \pm 0.39$ & $37.02 \pm 0.40$ & 0.84 \\
White blood cell $\left(\mathrm{x} 10^{9}\right)$ & $12.18 \pm 2.86$ & $11.93 \pm 1.95$ & 0.31 \\
Monocyte $\left(\mathrm{x} 10^{9}\right)$ & $0.79 \pm 0.36$ & $0.78 \pm 0.41$ & 0.27 \\
Lymphocyte $\left(\mathrm{x} 10^{9}\right)$ & $4.38 \pm 0.39$ & $4.28 \pm 0.63$ & 0.42 \\
Neutrophile granulocyte $\left(\mathrm{x} 10^{9}\right)$ & $8.04 \pm 1.89$ & $7.93 \pm 1.59$ & 0.48 \\
Blood glucose $(\mathrm{mmol} / \mathrm{l})$ & $8.05 \pm 2.34$ & $8.21 \pm 3.62$ & 0.26 \\
\hline
\end{tabular}

Table II. NIHSS scores in the two groups of patients (points, mean \pm SD).

\begin{tabular}{lcccc}
\hline Groups & No. & At admission & 1 day & 3 days \\
\hline A & 103 & $16.96 \pm 3.06$ & $16.39 \pm 3.56$ & $15.99 \pm 2.75$ \\
B & 103 & $17.05 \pm 2.97$ & $16.28 \pm 2.78$ & $15.01 \pm 2.04^{\mathrm{a}}$ \\
\hline
\end{tabular}

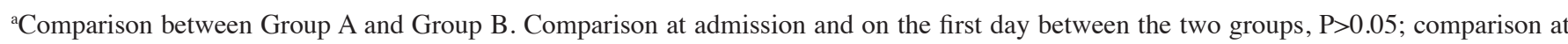
day 3 and 7 between the two groups, $\mathrm{P}<0.05$. SD, standard deviation.

Table III. Comparison of serum TNF- $\alpha$ concentrations in patients between the two groups (ng/ml, mean \pm SD).

\begin{tabular}{lcccc}
\hline Groups & No. & 1 day & 3 days & 7 days \\
\hline A & 103 & $3.1385 \pm 0.3759$ & $3.4984 \pm 0.5730$ & $2.8046 \pm 0.7028$ \\
B & 103 & $3.0037 \pm 0.4291$ & $2.8304 \pm 0.4826^{\mathrm{a}}$ & $1.1840 \pm 0.7820^{\mathrm{a}}$ \\
\hline
\end{tabular}

${ }^{\mathrm{a} C}$ Comparison of TNF- $\alpha$ contents in serum between the two groups $(\mathrm{P}<0.05)$. SD,standard deviation.

A

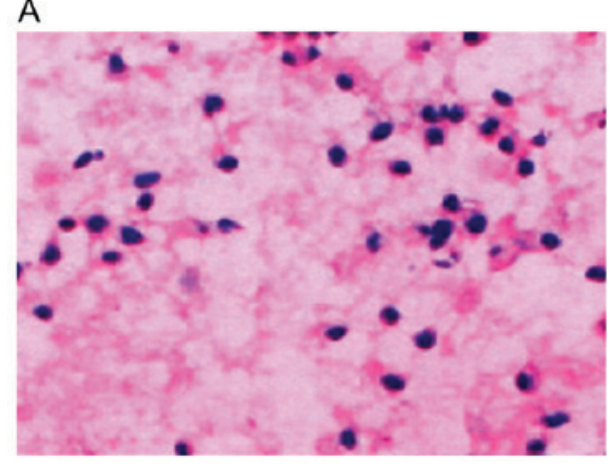

B

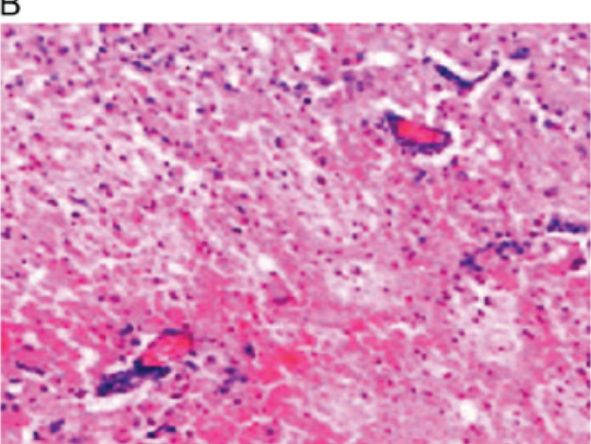

Figure 1. H\&E staining of tissue around the hematoma. (A) Loose tissue around the hematoma, extensive extravascular space, the gap around the nerve cells and glial cells (magnification, x200); (B) nerve cell body atrophy, karyopyknosis, Nissl's body disappearance and eosinophilic change in cytoplasm; a large number of neutrophils and lymphocytes around the hematoma (magnification, x100). H\&E, hematoxylin and eosin.

Immunohistochemical staining and $N F-\kappa B$ determination. Hematoxylin and eosin (H\&E) staining displayed loose tissue around the hematoma, extensive extravascular space, and a gap around the nerve cells and glial cells (Fig. 1A; H\&E, 


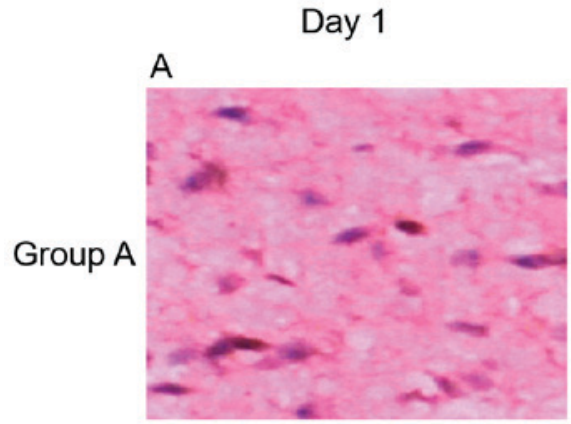

B

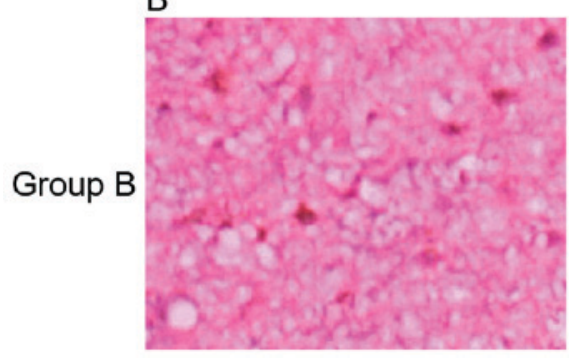

Day 3

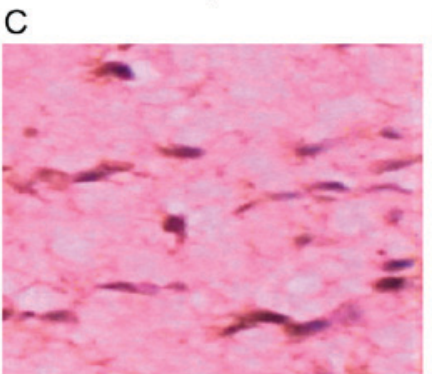

D

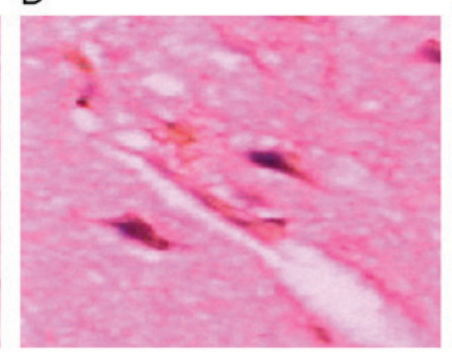

Day 7

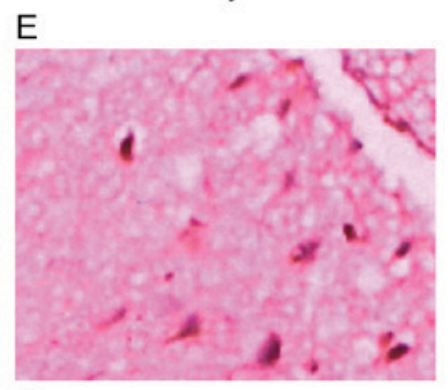

F

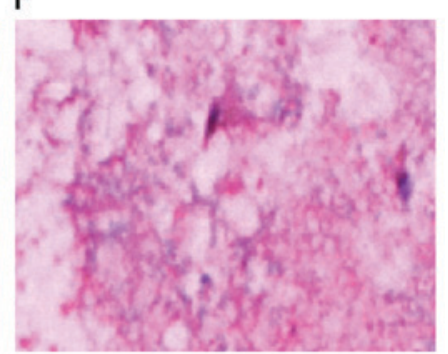

Figure 2. Detection of $\mathrm{NF}-\kappa \mathrm{B}$ expression in brain tissue around the hematoma using immunohistochemistry. (A) NF- $\mathrm{B}$ expression at day 1 in group $\mathrm{A}$ (magnification, $\mathrm{x} 400$ ); (B) NF- $\mathrm{B}$ expression at day 1 in group B (magnification, $\mathrm{x} 200$ ); (C) NF- $\mathrm{B}$ expression at day 3 in group A (magnification, $\mathrm{x} 400$ ); (D) $\mathrm{NF}-\kappa \mathrm{B}$ expression at day 3 in group B (magnification, $\mathrm{x} 400$ ); (E) NF- $\kappa \mathrm{B}$ expression at day 7 in group A (magnification, $\mathrm{x} 200$ ); (F) NF- $\kappa$ B expression at day 7 in group $\mathrm{B}$ (magnification, $\mathrm{x} 200)$. $\mathrm{NF}-\kappa \mathrm{B}$, nuclear factor- $\kappa \mathrm{B}$.

magnification, x200); nerve cell body atrophy, karyopyknosis, Nissl's body disappearance and eosinophilic change in cytoplasm; a large number of neutrophils and lymphocytes existed around the hematoma (Fig. 1B; H\&E, magnification, x100). Additionally, NF- $\mathrm{NB}$ was expressed in brain tissue around the hematoma in 206 patients, showing dynamic changes, which reached the peak at day 3 , and increased at days 1 and $7 . \mathrm{NF}-\kappa \mathrm{B}$ expression was reduced in patients in the mild hypothermia combined with minimally invasive hematoma evacuation group compared to that in the minimally invasive hematoma evacuation group. $\mathrm{NF}-\kappa \mathrm{B}$ was mainly expressed in the inflammatory cells, microglia and neural cells (Fig. 2).

\section{Discussion}

It has been demonstrated that mild hypothermia has a neuroprotective effect on patients with cerebral vascular disease, cerebral trauma and cerebral injury after cardiopulmonary resuscitation (7-9). The recent experiment and clinical study have indicated that mild hypothermia can also protect brain function after intracerebral hemorrhage via inhibiting immune inflammatory response, decreasing vascular permeability and reducing brain edema, stabilizing ion pump and inhibiting nerve excitability cascade reactions, reducing brain metabolism and other mechanisms. The cerebral protective effect of mild hypothermia was first applied in the study by Busto in 1987, and it has become the focus of brain protection again in recent years (10). The study revealed that the duration of mild hypothermia over $48 \mathrm{~h}$ can effectively reduce brain injury, and the longest duration is better when not more than $196 \mathrm{~h}$, temperature $32-35^{\circ} \mathrm{C}$. Currently, the studies of cardiopulmonary resuscitation showed that brain hypothermia induced by local cooling or combined with whole body cooling methods at the beginning of resuscitation that make patients reach target temperature $\left(34^{\circ} \mathrm{C}\right) 2 \mathrm{~h}$ earlier has a high safety and feasibility, and prognosis of the nervous system is significantly ameliorated $(11,12)$. Furthermore, no increase of complications such as heart disease, inflammatory lesion, and disturbance of blood coagulation, electrolyte abnormality, blood glucose abnormality or blood pressure abnormality has been found. Hence, we adopted whole body cooling plus local brain mild hypothermia therapy, decreasing brain temperature to $32.5-34.5^{\circ} \mathrm{C}$, lasting 3-7 days for mild hypothermia therapy, to treat patients with intracerebral hemorrhage after surgery of minimally invasive hematoma evacuation.

Clinical studies have revealed that during the acute cerebral hemorrhage period, body's inflammatory response is activated by central nervous system due to brain cell injury, thus producing a large number of inflammatory cytokines, such as TNF- $\alpha$ and IL-6; a great quantity of secretion and expression of inflammatory cytokines with proinflammatory effects is positively correlated with brain injury $(13,14)$. In a variety of inflammatory factors, TNF- $\alpha$ shows the earliest expression and the most release, while NF- $\kappa \mathrm{B}$ is an important pathway in the expression and progression of TNF- $\alpha$, IL- 6 and other inflammatory mediators, which is a crucial regulator of immune inflammatory reaction. Minimally invasive hematoma evacuation can decrease the hematoma volume, reduce the compression to surrounding brain tissue by hematoma, relieve cerebral edema, decrease intracranial pressure, reduce production of inflammatory factors and alleviate inflammatory injury, thus protecting brain cell function. Our experimental results displayed that after hemorrhage, TNF- $\alpha$ concentration in blood and $\mathrm{NF}-\kappa \mathrm{B}$ expression in brain tissue around hematoma changed in line with the illness; TNF- $\alpha$ content in serum was increased on the 1st day, reaching the peak on the 3rd day, and was the lowest on the 7th day; the same manner of change could be seen in $\mathrm{NF}-\kappa \mathrm{B}$ expression in brain tissue 
around the hematoma. TNF- $\alpha$ content and NF- $\kappa \mathrm{B}$ expression were lower in the mild hypothermia combined with minimally invasive hematoma evacuation group than those in the single minimally invasive surgery group at each time-point. NIHSS was the highest at admission and on the 1st day after treatment in the two groups of patients, and it was decreased with the treatment; the score was distinctly higher at 3 days than that at 7 days, which was consistent with the changes of TNF- $\alpha$ and NF- $\kappa$ B. NIHSS score was obviously lower in the mild hypothermia combined with minimally invasive hematoma evacuation group than that in the single minimally invasive hematoma evacuation group at 3 and 7 days.

In conclusion, mild hypothermia combined with minimally invasive hematoma evacuation can alleviate inflammatory response of brain tissue around hematoma in hypertensive intracerebral hemorrhage, thus effectively ameliorating and improving brain function. Our experimental results also confirmed the safety, effectiveness and feasibility of mild hypothermia combined with minimally invasive hematoma evacuation.

\section{Acknowledgements}

Not applicable.

\section{Funding}

The present study was financially supported by The Science and Technology Developing Fundation of Shanghai Pudong New Area (Shanghai, China)

\section{Availability of data and materials}

All data generated or analyzed during this study are included in this published article.

\section{Authors' contributions}

JiZ and QM designed the study, ZQi and JuZ collected the data, ZQu and $\mathrm{JuZ}$ analysed the data, JiZ and ZQu prepared the manuscript, JiZ and CW performed the experiments. All authors read and approved the final manuscript.

\section{Ethics approval and consent to participate}

This study was approved by the Ethics Committee of Shanghai Renji Hospital (Shanghai, China). Signed written informed consents were obtained from all participants before the study.

\section{Competing interests}

The authors declare that they have no competing interests.

\section{References}

1. Xu Y, Guo J, Liu X, Li J, Wang J and Hou L: Can herbal medicine cause hematoma enlargement of hypertensive intracerebral hemorrhage within $24 \mathrm{hrs}$ time window? A retrospective study of 256 cases from a single center in china. Evid Based Complement Alternat Med 2015: 868731, 2015.

2. Liu L and Yenari MA: Clinical application of therapeutic hypothermia in stroke. Neurol Res 31: 331-335, 2009.

3. Kollmar R, Staykov D, Dörfler A, Schellinger PD, Schwab S and Bardutzky J: Hypothermia reduces perihemorrhagic edema after intracerebral hemorrhage. Stroke 41: 1684-1689, 2010.

4. Broderick JP, Brott TG, Duldner JE, Tomsick T and Huster G: Volume of intracerebral hemorrhage. A powerful and easy-to-use predictor of 30-day mortality. Stroke 24: 987-993, 1993.

5. Schlegel D, Kolb SJ, Luciano JM, Tovar JM, Cucchiara BL, Liebeskind DS and Kasner SE: Utility of the NIH Stroke Scale as a predictor of hospital disposition. Stroke 34: 134-137, 2003.

6. Young FB, Weir CJ and Lees KR; GAIN International Trial Steering Committee and Investigators: Comparison of the National Institutes of Health Stroke Scale with disability outcome measures in acute stroke trials. Stroke 36: 2187-2192, 2005.

7. Abou-Chebl A, Sung G, Barbut D and Torbey M: Local brain temperature reduction through intranasal cooling with the RhinoChill device: Preliminary safety data in brain-injured patients. Stroke 42: 2164-2169, 2011.

8. Ma LL, Song L, Yu XD, Yu TX, Liang H and Qiu JX: The clinical study on the treatment for acute cerebral infarction by intra-arterial thrombolysis combined with mild hypothermia. Eur Rev Med Pharmacol Sci 21: 1999-2006, 2017.

9. Martin-Schild S, Hallevi H, Shaltoni H, Barreto AD, Gonzales NR, Aronowski J, Savitz SI and Grotta JC: Combined neuroprotective modalities coupled with thrombolysis in acute ischemic stroke: A pilot study of caffeinol and mild hypothermia. J Stroke Cerebrovasc Dis 18: 86-96, 2009.

10. Busto R, Dietrich WD, Globus MY, Valdes I, Scheinberg P and Ginsberg MD: Small differences in intraischemic brain temperature critically determine the extent of ischemic neuronal injury. J Cereb Blood Flow Metab 7: 729-738, 1987.

11. Tsai MS, BarbutD, Wang H, Guan J, Sun S, Inderbitzen B,Weil MH and Tang W: Intra-arrest rapid head cooling improves post resuscitation myocardial function in comparison with delayed post resuscitation surface cooling. Crit Care Med 36: 434-439, 2008.

12. Guan J, Barbut D, Wang H, Li Y, Tsai MS, Sun S, Inderbitzen B, Weil MH and Tang W: A comparison between head cooling begun during cardiopulmonary resuscitation and surface cooling after resuscitation in a pig model of cardiac arrest. Crit Care Med 36: 428-433, 2008

13. Nimmo AJ and Vink R: Recent patents in CNS drug discovery: The management of inflammation in the central nervous system. Recent Patents CNS Drug Discov 4: 86-95, 2009.

14. Werner $C$ and Engelhard K: Pathophysiology of traumatic brain injury. Br J Anaesth 99: 4-9, 2007. International (CC BY-NC-ND 4.0) License.

\section{Consent for publication}

Not applicable. 